\title{
Compassion Focused Therapy Groups: Evidence from Routine Clinical Practice
}

\author{
James McManus, Zoe Tsivos, Sarah Woodward, Janelle Fraser and Rosalyn Hartwell \\ Greater Manchester Mental Health NHS Trust, Manchester, UK \\ Address for correspondence: Dr James McManus, Clinical Psychologist, Department of Clinical Psychology, \\ North Manchester General Hospital, Delaunays Road, Crumpsall, Manchester M8 5RB, UK. \\ Email: james.mcmanus@gmmh.nhs.uk
}

\begin{abstract}
Objectives. Psychological therapy groups for people in adult mental health services can relieve waiting list pressures and potentially reduce stigma and social isolation. Compassion-focused therapy (CFT) focuses on shame and self-criticism. The aim of this study was to evaluate a transdiagnostic CFT group.

Design. Quantitative and qualitative data were obtained from 13 people who completed the group.

Methods. Participants completed a range of pre- and post-group self-report outcome measures that assess self-criticism and self-compassion.

Results. Statistically significant improvements were found on all measures used, suggesting that attending the CFT group did result in meaningful changes. People who completed the group also provided positive feedback about the experience.

Conclusions. The results suggest that running CFT groups is feasible and acceptable to clients with a range of psychiatric diagnoses as part of their care from community mental health teams. People who completed the group demonstrated significant improvements. A proportion of people did not complete the group, and more research is required about the reasons for this. Limitations of this study are considered together with future directions for research into CFT.
\end{abstract}

Keywords: compassion; group; therapy; community; mental; health; NHS

Compassion-focused therapy (CFT) is an increasingly popular form of psychological therapy and is considered part of the third wave of cognitive-behavioural therapies (CBT). While there are some shared features with CBT (e.g., re-evaluating thoughts, focusing on behavioural change, using imagery), CFT also draws on attachment theory, evolutionary theory, neuro-scientific evidence, and an understanding of compassion that is rooted in Buddhist philosophy. It is a multimodal therapy, with a particular focus on shame and self-criticism (Gilbert, 2014).

As a relatively new form of therapy, the evidence base is emerging. To date, there are a small number of published randomised controlled trials (RCT) using clinical samples, including psychosis (Braehler et al., 2012), binge-eating disorder (Kelly \& Carter, 2015), acquired brain injury (O’Neill \& McMillan, 2012), depression and anxiety (Noorbala, Borjali, Ahmadian-Attari, \& Noorbala, 2013) and post-traumatic stress disorder (Beaumont, Galpin, \& Jenkins, 2012). These studies have demonstrated promising results across disorders and in transdiagnostic groups. In particular, CFT offers promise in targeting the common psychological problems of shame and self-criticism.

In a feasibility study for people with a diagnosis of psychosis, Braehler et al. (2012) compared a CFT group intervention with treatment as usual (TAU) for 40 participants. After 16 sessions, the CFT intervention group was associated with greater observed clinical improvement (using a scale rated by a therapist blind to treatment condition), significant increases in compassion scores, and significant reductions in measures of depression and perceived social marginalisation. The CFT group was associated with no adverse events, low attrition (18\%), and high acceptability. 
Other studies evaluating CFT with people with severe and enduring mental health problems have tended to report data from case and observational studies. Mayhew and Gilbert (2008) reported on a case series of compassionate mind training (a key feature of CFT) for three individuals hearing malevolent voices. The study found that after 12 weekly sessions, all participants reported fewer malevolent and fewer persecuting voices, and two of the three heard more reassuring voices.

There has also been a focus on evaluating group CFT interventions for this population. Judge, Cleghorn, McEwan, and Gilbert (2012) provided CFT groups for clients under the care of community mental health teams (CMHTs). Data were gathered from 27 participants with diagnoses of schizophrenia or bipolar disorder. Significant improvements were reported on self-report measures of shame, self-criticism, submissive behaviour, depression and anxiety. Lucre and Corten (2013) reported results from a CFT group for eight participants with a diagnosis of personality disorder. Results here were more mixed, with significant improvements reported on measures of social comparisons, external shame, hated self, reassuring self, depression and stress, but no significant change on measures of submissive behaviour, inadequate self and anxiety.

In addition to these community groups, other reports have provided data from groups in in-patient settings. Heriot-Maitland, Vidal, Ball, and Irons (2014) provided a four-session modular CFT group and reported data from 57 attendees, demonstrating significant reductions in distress ratings and significant increases in calmness ratings. The authors concluded that open, trans-diagnostic groups were feasible and well received on acute inpatient wards. Laithwaite et al. (2009) reported on a 10-week CFT group in a high-secure hospital. Data from 19 adult males with a diagnosis of schizophrenia or bipolar disorder showed significant improvements on measures of depression and self-esteem, moderate change on measures of social comparisons, and a small magnitude of change for measures of shame.

In a systematic review of the published evidence for CFT, Leaviss and Uttley (2015) concluded that CFT shows promise as an intervention for mood disorders, particularly for people with high levels of self-criticism. However, it was also noted that more high-quality trials are needed, including RCTs that compare CFT to TAU and also to other forms of psychological therapy. Mixed-methods research with long-term follow-up would also be an important area for future research in this area.

The aim of this study was to add to the emerging evidence base for CFT by reporting data from a service evaluation of a trans-diagnostic CFT group for people with severe and enduring mental health problems working with community mental health teams (CMHTs). Given the large numbers of referrals to the clinical psychology service within the CMHTs, group interventions can represent an efficient use of therapist time and also provide an alternative treatment choice for clients, with the additional potential benefits of group therapy (Yalom \& Leszcz, 2005). Staff working in clinical psychology services had noted high numbers of referrals for people presenting with emotion regulation problems and high levels of shame and self-criticism. Therefore, it was decided to provide a CFT group as part of the range of therapy intervention options available and to evaluate the outcomes of this in routine clinical practice.

\section{Method}

\section{Participants}

Participants were adults receiving a service from six CMHTs in a large city. Care coordinators were asked to identify people in their caseload with high levels of shame and self-criticism, regardless of diagnosis, to refer for the group. People referred for the group intervention were then offered an assessment appointment to provide information about the group and consider suitability (i.e., that they were reporting high levels of shame and self-criticism, and were able and willing to participate in a therapy group).

\section{Intervention}

The CFT group intervention was developed by the authors following completion of training in CFT and based on published manuals (Braehler, Harper, \& Gilbert, 2013; Gilbert, 2010; Welford, 2012). 
Table 1. Content of the CFT Group Sessions

\begin{tabular}{|c|c|}
\hline Session & Summary of session themes \\
\hline 1 & $\begin{array}{l}\text { Psycho-education: What is CFT? Evolution model, three emotion regulation systems, defining shame, } \\
\text { self-criticism and compassion }\end{array}$ \\
\hline 2 & Motivation: Stages of change and breaking through fears and blocks \\
\hline 3 & Mindfulness and soothing rhythm breathing \\
\hline 4 & Drawing it all together - CFT formulation \\
\hline 5 & Individual session - CFT formulation \\
\hline 6 & Imagery: Safe place imagery, compassionate colour \\
\hline 7 & Imagery: Compassion for others \\
\hline 8 & Imagery: Developing your compassionate self \\
\hline 9 & Imagery: Your compassionate ideal self \\
\hline 10 & Compassionate thinking (1) \\
\hline 11 & Compassionate thinking (2) \\
\hline 12 & Compassionate letter writing (1) \\
\hline 13 & Compassionate letter writing (2) \\
\hline 14 & Compassionate behaviour (1) \\
\hline 15 & Compassionate behaviour (2) \\
\hline 16 & Final session and review \\
\hline
\end{tabular}

The content of the group is described in Table 1. Four different groups were delivered across the three areas of the city covered by the CMHTs. Each group was delivered by two of the authors, all of whom are clinical psychologists or trainee clinical psychologists. Each group followed the same format of 16 sessions, meeting weekly for two hours. Participants were also asked to complete homework practice in between sessions and were provided with an audio CD (produced by the authors) of the relevant mindfulness and imagery exercises to support this.

\section{Self-Report Measures}

Participants completed the following self-report measures pre- and post-group (i.e., in the initial assessment appointment and at the final group session). These measures were selected to be in line with previous research and because they focus on outcomes relevant to CFT, namely reduced selfcriticism and increased self-compassion.

Forms of Self-Criticising/Attacking and Self-Reassuring Scale (FSCRS; Gilbert, Clarke, Kempel, Miles, \& Irons, 2004)

The FSCRS measures self-criticism and the ability to self-reassure. It is a 22 -item scale that measures different ways people think and feel about themselves when things go wrong for them. The items make up three components: inadequate self, which focuses on a sense of personal inadequacy; hated self, which focuses on the desire to hurt or persecute the self; and reassured self, which focuses on the ability to self-reassure. The inadequate self-subscale has excellent internal consistency $(\alpha=0.90)$, and good internal consistency was reported for the hated self $(\alpha=0.86)$ and self-reassurance $(\alpha=0.86)$ subscales (Gilbert et al., 2004).

Functions of Self-Criticising/Attacking Scale (FSCS; Gilbert et al., 2004)

The FSCS measures the functions of self-criticism, that is, the reasons that people think they selfcriticise and self-attack. Factor analysis suggests two different functions: self-correction (trying to 
improve the self and stop the self from making mistakes) and self-persecution (expressing anger and wanting to harm the self). The scale has excellent internal consistency, $(\alpha=0.92$; Gilbert et al., 2004).

Other As Shamer Scale (OAS; Goss, Gilbert, \& Allan, 1994)

The OAS evaluates the extent to which people experience others as critical and rejecting. It consists of 18 items rated on a 5-point scale according to the frequency of evaluations about how others judge the self. The OAS has high internal consistency $(\alpha=0.92-0.96$; Goss et al., 1994).

\section{Self-Compassion Scale (SCS; Neff, 2003)}

The SCS assesses trait levels of self-compassion. The scale includes items that measure how often people respond to feelings of inadequacy or suffering with self-kindness, self-judgment common humanity, isolation, mindfulness, and over-identification. All subscales have good internal consistency, self-kindness $(\alpha=0.78)$, self-judgement $(\alpha=0.77)$, common humanity $(\alpha=0.80)$, isolation $(\alpha=$ $0.79)$, mindfulness $(\alpha=0.75)$ and over-identified $(\alpha=0.81)$. The global scale has excellent internal consistency $(\alpha=0.92$; Neff, 2003).

\section{Mental Health Confidence Scale (MHCS; Carpinello et al., 2000)}

The MHCS is designed to assess the self-efficacy beliefs of people dealing with mental disorders, particularly how confident they are about their ability to deal with those things that commonly influence their lives, using a three-factor structure of optimism, coping and self-efficacy. Results of confirmatory factor analyses support the three-factor structure and suggest that the scale is reliable (Carpinello, Knight, Markowitz, \& Pease, 2000).

In addition to self-report measures, participants were also asked for qualitative feedback at an individual follow-up appointment.

\section{Results}

\section{Participants}

Twenty-seven participants were offered the CFT group therapy intervention. Of these, 14 people either did not attend or dropped out prior to completion. A total of 13 people completed the group and provided pre- and post-outcome data (6 female, 7 male; age range 23-67, mean age 43).

The primary diagnoses, obtained from the clinical records as part of routine clinical practice, for those completing the group included schizophrenia $(n=5)$, bipolar disorder $(n=4)$, depressive disorder $(n=3)$, and emotionally unstable personality disorder $(n=1)$. Four participants also had a comorbid diagnosis: panic disorder $(n=1)$, generalised anxiety disorder $(n=1)$, alcohol dependence $(n=1)$, and grief reaction $(n=1)$.

There was no follow-up data from those who did not attend or dropped out of therapy, although all such participants were contacted and offered a reassessment to consider alternative therapy options.

\section{Self-Report Measures}

The data were analysed with SPSS using the Wilcoxon signed-rank test. This is a non-parametric test that looks for differences between two related samples. The results are given in Table 2.

The results show that significant changes between start and end of the group were found on all measures used. There was only one subscale that did not show significant change (the reassure self subscale of the FSCRS). The scores on all subscales did move in the direction of improvement (i.e., scores increased on the reassure self-subscale of the FRCRS, the self-kindness, common humanity, and mindfulness subscales of the SCS, and on the MHCS; scores decreased on the inadequate self and hated self subscales of the FSCRS, the self-judgement, isolation, and over-identified subscales of the SCS). 
Table 2. Changes in Scores on Self-Report Measures Pre- and Post-Therapy Group

\begin{tabular}{|c|c|c|c|c|c|}
\hline Scale & Subscale & $\begin{array}{l}\text { Median score } \\
\text { pre-group }\end{array}$ & $\begin{array}{l}\text { Median score } \\
\text { post-group }\end{array}$ & z score & $p$ value \\
\hline \multirow[t]{3}{*}{ FSCRS } & Inadequate self & $27(S D=6.38)$ & $14(S D=9.25)$ & -2.13 & .03 \\
\hline & Hated self & $12(S D=4.94)$ & $7(S D=5.04)$ & -2.31 & .02 \\
\hline & Reassure self & $14(S D=5.53)$ & $19(S D=6.69)$ & -1.81 & .07 \\
\hline OAS & Total & $40(S D=15.59)$ & $17(S D=19.50)$ & -2.69 & .01 \\
\hline \multirow[t]{7}{*}{ SCS } & Total & $53(S D=10.15)$ & $67(S D=23.66)$ & -2.56 & .01 \\
\hline & Self-kindness & $1.8(S D=0.72)$ & $2.6(S D=1.11)$ & -2.23 & .03 \\
\hline & Self-judgement & $4(S D=0.85)$ & $3(S D=1.18)$ & -2.04 & .04 \\
\hline & $\begin{array}{l}\text { Common } \\
\text { humanity }\end{array}$ & $2.75(S D=0.73)$ & $3.25(S D=0.97)$ & -1.97 & .05 \\
\hline & Isolation & $3.75(S D=0.84)$ & $2.5(S D=1.32)$ & -2.41 & .02 \\
\hline & Mindfulness & $2.25(S D=0.61)$ & $3.25(S D=0.83)$ & -2.36 & .02 \\
\hline & Overidentified & $4(S D=0.73)$ & $2.5(S D=1.32)$ & -2.71 & .01 \\
\hline MHCS & Total & $47(S D=15.44)$ & $65(S D=10.48$ & -2.27 & .02 \\
\hline
\end{tabular}

\section{Qualitative Feedback}

Participants who completed the group were asked to provide written feedback on their experience. All participants gave positive feedback about attending the sessions; a selection of these comments can be found below:

I have just completed the Compassion Focussed Therapy Group. At first I was really nervous and apprehensive. But after the first few weeks I found it was fine and in fact has changed my life completely. This has been the longest spell of being well and staying out of hospital. I found the compassionate imagery and compassionate letter writing really useful techniques to help maintain my mental health. I would recommend this group to anyone with mental health difficulties.

I would really recommend Compassion Focused Therapy. It is a big time commitment but it is so worth it. It has made a huge difference to my life. It's not easy to make changes but the group has been so supportive throughout. I was really unsure about group instead of individual therapy but I now feel it a fantastic therapeutic approach. The psychologists are great. Keeping up with the strategies is hard but the support group is there to keep it going after the 16 weeks have ended.

I have learned exercises that I use in everyday life and have helped me overcome bad days. Thank you for all your help, I will always use my exercises.

I enjoyed the group sessions. I've learned a lot about how to feel with my emotions and I have learned to accept myself for who I am.

I have learned to be more mindful and the group has helped me manage my panic attacks better.

The course and the people I met have been an extremely good experience in compassion ... thank you for providing a course that perhaps every human should experience.

CFT has given me access to emotions I was consciously cut off from and a method to be more gentle and considerate to myself. With practice I will be able to deal with the difficult emotional 
punishments I have inflicted on myself for many years and it will stand me in good stead for whatever life throws at me in the future. CFT does not just bring compassion for the self, by extension such therapy extends into society in general. By being able to deal with real or imagined threats with compassion I hope I will be better able to interact with people with both understanding and compassion

\section{Discussion}

This was an evaluation study of a CFT group run in NHS community mental health teams in England. The aim was to consider the acceptability and effectiveness of this group intervention for this client group. The results have shown significant improvements for those people who completed the group on a number of relevant outcome measures. People who completed this group reported significant improvements in self-compassion, reductions in self-criticism and shame, and improvements in selfconfidence and self-efficacy. People who completed the group also provided positive feedback about both the content of the group and the group process, often highlighting the support of other group members as very important in feeling less alone.

However, it is important to note that that out of 27 individuals offered the group, only 13 (48\%) completed the group, and the remaining individuals were lost to follow-up. This is similar to the dropout rate reported in other studies (e.g., Mayhew \& Gilbert, 2008). Some people did not attend any sessions, whereas others did attend for at least one group session and then dropped out. It has been noted in previous research that some people report finding CFT work difficult, and indeed working on fears and blocks is a core part of the therapy (Gilbert, 2014). Previous research has noted participants finding it difficult to hold on to more than a fleeting compassionate image (Gilbert \& Irons, 2004), difficulties generating an image that is not also self-critical (Mayhew \& Gilbert, 2008), and a range of fears or blocks, including thoughts that self-compassion is self-indulgent or destructive (Lucre \& Corten, 2013).

It was apparent when facilitating the group sessions that some people did find the experience of being in the group and discussing self-compassion to be very difficult. This would be predicted by the model, which highlights fears and blocks to compassion as the central part of the work. It may be inevitable when working with this model that some clients will experience their 'threat' mode and related attachment system to be activated, and this may be more difficult for both clients and facilitators to manage in a group setting. This highlights the importance of a thorough assessment of suitability for the group, and it is recommended that future participants may benefit from more individual preparation for the work prior to entering the group. Certainly, the individual formulation session offered at session 5 was found to be very helpful, and provided an opportunity to address individual fears and concerns in more detail, as well as ensuring group content had been fully understood.

The therapy groups in this study were open to people under the care of the CMHT. This included individuals with complex, long-standing mental health problems, risk issues, and diagnoses including psychosis and personality disorder. This study adds to the existing literature (Braehler et al., 2013; Heriot-Maitland et al., 2014; Lucre \& Corten, 2013) that shows that CFT groups can be used with this population and can produce significant improvements. Furthermore, the study shows some feasibility and acceptability for providing trans-diagnostic groups that focus on common problems such as shame and self-criticism. Such groups have the potential to reduce stigma and provide an alternative to focusing on psychiatric diagnoses. Future research should focus on the extent to which these improvements contribute to everyday functioning and recovery, evidenced by increased achievement of personal goals and reduced need for support from mental health services. It will also be important to consider the group of people who were not able to complete the group work and identify the reasons for this and other ways in which these people can be supported.

Acknowledgments. This research received no specific grant from any funding agency, commercial or not-for-profit sectors.

Declaration of interest. None. 


\section{References}

Beaumont EA, Galpin AJ and Jenkins PE (2012) 'Being kinder to myself: A prospective comparative study, exploring posttrauma therapy outcomes, for two groups of clients, receiving either cognitive behaviour therapy or cognitive behaviour therapy and compassionate mind training. Counselling Psychology Review 27, 31-43.

Braehler C, Gumley A, Harper J, Wallace S, Norrie J and Gilbert P (2012) Exploring change processes in compassion focused therapy: Results of a feasibility randomised controlled trial. British Journal of Clinical Psychology 52, $199-214$.

Braehler C, Harper J and Gilbert P (2013) Compassion focused group therapy for recovery after psychosis. In C Steel (Ed.), Cognitive behavior therapy for schizophrenia: Evidence-based interventions and future directions (pp. 236-266). West Sussex, UK: Blackwell.

Carpinello SE, Knight EL, Markowitz FE and Pease EA (2000) The development of the mental health confidence scale: A measure of self-efficacy in individuals diagnosed with mental disorders. Psychiatric Rehabilitation Journal 23, $236-243$.

Gilbert P (2010) Training our minds in, with and for compassion: An introduction to concepts and compassion-focused exercises. Retrieved from http://wtm.thebreathproject.org/wp-content/uploads/2016/03/COMPASSION-HANDOUT.pdf

Gilbert P (2014) The origins and nature of compassion focused therapy. British Journal of Clinical Psychology 53, 6-41.

Gilbert P and Irons C (2004) A pilot exploration of the use of compassionate images in a group of self-critical people. Memory 12, 507-516.

Gilbert P, Clarke M, Kempel S, Miles JNV and Irons C (2004) Criticising and reassuring oneself: An exploration of forms, style and reasons in female students. British Journal of Clinical Psychology 43, 31-50.

Goss K, Gilbert P and Allan S (1994) An exploration of shame measures - I: the other as shamer scale. Personality and Individual Differences 17, 713-717.

Heriot-Maitland C, Vidal JB, Ball S and Irons C (2014) A compassion-focused therapy group approach for acute inpatients: Feasibility, initial pilot outcome data, and recommendations. British Journal of Clinical Psychology 53, 78-94.

Judge L, Cleghorn A, McEwan K and Gilbert P (2012) An exploration of group-based compassion focused therapy for a heterogeneous range of clients presenting to a community mental health team. International Journal of Cognitive Therapy 5, 420-429.

Kelly AC and Carter JC (2015) Self-compassion training for binge eating disorder: A pilot randomized controlled trial. Psychology and Psychotherapy: Theory, Research and Practice 88, 285-303.

Laithwaite HL, O'Hanlon M, Collins P, Doyle P, Abraham L, Porter S and Gumley A (2009) Recovery after psychosis (RAP): A compassion focused programme for individuals residing in high security settings. Behavioural and Cognitive Psychotherapy 37, 511-526.

Leaviss J and Uttley L (2015) Psychotherapeutic benefits of compassion-focused therapy: An early systematic review. Psychological Medicine 45, 927-945.

Lucre KM and Corten N (2013) An exploration of group compassion-focused therapy for personality disorder. Psychology and Psychotherapy: Theory, Research and Practice 86, 387-400.

Mayhew SL and Gilbert P (2008) Compassionate mind training with people who hear malevolent voices: A case series report. Clinical Psychology and Psychotherapy 15, 113-138.

Neff KD (2003) The development and validation of a scale to measure self-compassion. Self and Identity 2, 223-250.

Noorbala F, Borjali A, Ahmadian-Attari MM and Noorbala AA (2013) Effectiveness of compassionate mind training on depression, anxiety, and self-criticism in a group of Iranian depressed patients. Iranian Journal of Psychiatry 8, 113-117.

O’Neill M and McMillan TM (2012) Can deficits in empathy after head injury be improved by compassionate imagery? Neuropsychological Rehabilitation 22, 836-851.

Welford M (2012) The compassionate mind approach to building your self-confidence using compassion focused therapy. London, UK: Robinson.

Yalom ID and Leszcz M (2005) The theory and practice of group psychotherapy. New York, NY: Basic Books.

Cite this article: McManus J, Tsivos Z, Woodward S, Fraser J, Hartwell R (2018). Compassion Focused Therapy Groups: Evidence from Routine Clinical Practice. Behaviour Change 35, 167-173. https://doi.org/10.1017/bec.2018.16 